\title{
Cluster arithmetic made simpler
}

Clusters of small numbers of identical atoms are all the rage, but the calculation of their properties taxes even large computers. That is why a simpler method deserves a welcome, but it also has heuristic value.

THE cluster business, the study of small numbers of identical atoms held together by a combination of chemical bonds and electrostatic forces, is booming away, and for a variety of reasons. As always, new techniques have had a powerful influence, notably those allowing for the selfassembly of clusters from the ingredients of well-cooled atomic beams. A further incentive is the prospect that atomic clusters may have important practical advantages, as catalysts for example, or possibly as sub-components of electronic devices. Evidently there are prizes to be won.

But the cluster business has also turned up surprises, not least that the configurations of small clusters are often strikingly different from what would be expected from the interatomic configurations in the bulk solids. Both the four-atom and sixatom clusters of lithium are planar structures; in $\mathrm{Li}_{4}$, the atoms are at the vertices of a rhombus, in $\mathrm{Li}_{6}$, three atoms are at the vertices of an equilateral triangle and the others at the mid-points of the three sides. Why should these unexpected structures be stable? And, more generally, what can be said about the transition from small clusters to the interatomic configuration of bulk solids?

These are questions from which Osamu Sugino and Hiroshi Kamimura of the University of Tokyo embark on a simplified way of calculating the stability of small clusters (Phys. Rev. Lett 65 2,696; 1990). Their starting-point is a set of data for the stability of small lithium-atom clusters. In the process, they describe a novel kind of bond between atoms, a "glue bond" as they call it.

That the cluster business needs a simplified method of calculation is not disputed. The techniques of computational chemistry may be used to calculate the properties of an arbitrary atom cluster, which for these purposes is simply another kind of molecule. For lithium clusters, for example, in which each atom is a singly charged helium-like core with a single external electron, it would be reasonable to begin with the assumption that there is one potentially bonding electron on each atom. For $\mathrm{Li}_{4}$, it would then suffice to calculate the energy of the four-electron system for all possible configurations of the four nuclear cores.

The snag, as Sugino and Kamimura note, is that even those with access to supercomputers are lucky to win the time required for comprehensive calculations. For each cluster is intrinsically more difficult than a molecule. In principle, the configuration and even its symmetry may not be known, while telling what is the optimum configuration depends on a comparison between that and all the others possible. The calculation of clusters with large numbers of atoms is, for practical purposes and at least for the time being, only exceptionally feasible.

So is there a simpler way? Sugino and Kamimura go back to an earlier attempt at generalization by Mark $\mathrm{H}$. McAdon and William A. Goddard (Phys. Rev. Lett 55, $2,563 ; 1985)$, also based on calculations of lithium clusters. Their objective was to construct configurational rules on the basis of accurate calculations of a few simple lithium clusters in a few simple configurations.

One of their more striking findings was that the distribution of electron density in, say, a square $\mathrm{Li}_{4}$ configuration is concentrated about the sides of the square, or symmetrically about the mid-points of the interatomic bonds to be precise, and not at the atoms themselves. Those who choose to regard this as a hint of the electron delocalization found in the conduction band of an electronic solid, a metal for example, will not be mistaken.

McAdon and Goddard also found that each of the electron orbitals in $\mathrm{Li}_{4}$ is occupied by a single electron only, with the spins of neighbouring electrons paired (in the sense of being antiparallel) in pairs. The distortion of the square into a rhombus is advantageous because of the physical repulsion between parallel (or Pauli-excluded) electrons. Another way of putting this is to say that the acute angle of the rhombus (measured at about $60^{\circ}$ ) includes two paired electrons in a roughly spherical orbital filling that triangular half of the rhombus.

What Sugino and Kamimura have now done, in the hope of simplying accurate calculations of lithium clusters, is to embark on quantum chemistry with electron orbitals which more accurately represent this kind of electron distribution. They represent them as ellipsoids centred on moveable points and, allowing for the electrostatic repulsion between the helium-like cores of the lithium atoms, carry through a calculation to determine not only the parameters defining the ellipsoids but the positions of the nuclei and the points around which the different elec- trons are centred. These are what they call their glue-bonds.

The outcome of the calculations is impressive. First, the stability (or the negative energy) of lithium clusters is accurately reproduced up to the limit of strict $a b$ initio calculations based on selfconsistent field optimization. $\mathrm{Li}_{\mathrm{s}}$, for example, emerges (as it should) as one of the most stable clusters. It is a curious structure, which may be considered as two $\mathrm{Li}_{4}$ rhombi, one above the other, which are puckered into boat shapes in opposite directions with one turned through $90^{\circ}$. There are four glue-bonds, each occupied by a pair of electrons, which are themselves arranged tetrahedrally (whence, say the authors, the stability).

The authors carry their calculations beyond $\mathrm{Li}_{14}$, the most complicated cluster to yield to self-consistent field treatment, as far as $\mathrm{Li}_{36}$. They conclude that there are "magic numbers" of stability at clusters of $8,14,18,20,26,30$ and 34 atoms. although, with almost half the even numbers between 8 and 36 in the list, there may be too much magic for some tastes. The numbers 8,20 and 30 might be a more prudent choice, to judge from the results of the calculations.

What does this mean for the transition from cluster to bulk solid? The optimum configuration for any cluster is evidently a trade-off between the electrostatic energy and the electron exchange or Pauliexclusion energy. The case of $\mathrm{Li}_{4}$ illustrates that an increase of the former on distortion of a symmetrical structure may be more than offset by a decrease of the latter involving the physical separation of glue-bonds from each other.

What Sugino and Kamimura argue is that there comes a point, as clusters become larger, at which it is no longer energetically favourable to place all the atoms on the outside surface of an open network. Then, there is less scope for geometrical distortion of the kind required if exchange energy is to be minimized, so that magic numbers melt more completely into the background.

The calculations show that this transition to bulk constitution must be under way at $\mathrm{Li}_{26}$, the least energetic form of which has glue-bonds in a pattern with icosahedral symmetry, which in turn implies that one of them is enclosed within a shell of all the others. The cluster is not yet a metal, but nearly so.

John Maddox 